\title{
Prebiotic-chemistry inspired polymer coatings for biomedical and material science applications
}

\author{
Helmut Thissen ${ }^{1}$, Aylin Koegler ${ }^{1}$, Mario Salwiczek ${ }^{1}$, Christopher D Easton ${ }^{1}$, Yue $\mathbf{Q u}^{2}$, Trevor Lithgow ${ }^{2}$ and \\ Richard A Evans ${ }^{1}$ \\ In the field of prebiotic chemistry, hydrogen cyanide (HCN)-derived polymers have been studied for many years as a possible \\ source of the precursors that provide the building blocks for proteins as well as nucleic acids, and they have also been \\ associated with the origin of life. The HCN trimer, aminomalononitrile (AMN), polymerizes to give a brown complex nitrogenous \\ polymer. We report the one-step polymerization-deposition of AMN as a simple generic surface-coating method and as an \\ application of prebiotic chemical research to material science. We found that this polymerization, carried out in buffered \\ aqueous solutions, can be used to coat a wide range of organic and inorganic substrate materials. The robust, non-cytotoxic \\ coatings also provide for excellent cell attachment, suggesting potential biomedical applications. Furthermore, the coating \\ chemistry allows for the immobilization of other compounds, including metals, both during coating formation or by performing \\ secondary immobilization reactions.
}

NPG Asia Materials (2015) 7, e225; doi:10.1038/am.2015.122; published online 13 November 2015

\section{INTRODUCTION}

Prebiotic chemistry or chemical evolution is the study of the chemistry required for producing the key molecules that eventually led to the origin of life. ${ }^{1-4}$ Miller ${ }^{5,6}$ initiated the era of experimental prebiotic chemistry with his famous spark experiment using methane, hydrogen and ammonia that produced complex organic compounds such as amino acids. Matthew's and Ferris' separate studies of HCN polymerization chemistry, as well as Sagan's and Khare's study of tholins, also involved the facile production of complex, highly colored organic polymers. $^{7-15}$ They have also been considered as a potential source of amino acids, nucleobases and peptides on earth and appear to be common throughout our solar system. For example, these materials have been proposed to be the source of the distinctive orange, redbrown and black colors of comets and the atmosphere of planets and moons such as Saturn, Jupiter and Titan and carbonaceous meteorites, indicating their abundance in our solar system and the universe, respectively. ${ }^{10,14-16}$

However, the focus of prebiotic chemistry has always been on understanding the chemical origin of life and thus has remained highly fundamental research. ${ }^{4}$ The application of prebiotic chemistry and specifically prebiotic polymers to material science has not been reported.

The polymers examined in the prebiotic field offer significant opportunities for practical exploitation because of the ease of their production and the scope for tuning material and chemical properties through the reaction conditions used and the choice of monomers. At the same time, there is an increasing need for methods that provide tailored surface properties while maintaining the bulk material properties of the underlying substrates. Surface modification methods span a wide variety of applications ranging from solar cells to implantable medical devices. The need for surface modification in the context of biomedical applications is especially important because the biological response is often determined by biointerfacial interactions. ${ }^{17}$ However, there are very few simple generic aqueous coating methods that are both robust and versatile as well as easily applicable over a range of substrate materials. ${ }^{18,19}$ Here, we report our findings in regard to the use of the polymerization of aminomalononitrile (AMN) as a simple general coating and surface modification method (Figure 1a)..$^{20}$

AMN, along with iminoacetonitrile, has received attention as an intermediate in the polymerization of hydrogen cyanide as well as a highly reactive synthon in heterocyclic organic synthesis. ${ }^{7,11,13,21-23}$ It is formally a trimer of hydrogen cyanide and spontaneously polymerizes when commercially available $p$-toluenesulfonate is neutralized (Figure $1 \mathrm{~b}){ }^{24}$ It allows easy access to $\mathrm{HCN}$ polymers under very mild and controllable conditions without the need to use $\mathrm{HCN}$ itself. ${ }^{24,25}$ The extremely adhesive polymer produced is structurally very complex. Hydrolysis experiments of the polymers result in the observation of amino acids with particularly significant amounts of glycine and polycyclic nitrogenous compounds such as purines and imidazole derivatives (Supplementary Figure S1). ${ }^{9,11,24-30}$ Because of these properties, we investigated the AMN polymer as a candidate for a new generic coating method that may be particularly useful in

${ }^{1}$ Manufacturing Flagship, Commonwealth Scientific and Industrial Research Organisation (CSIRO), Clayton, VIC, Australia and ${ }^{2}$ Department of Microbiology, Monash University, Melbourne, VIC, Australia

Correspondence: Dr RA Evans or Dr H Thissen, Manufacturing Flagship, Commonwealth Scientific and Industrial Research Organisation (CSIRO), Bayview Avenue, Clayton, VIC 3168, Australia.

E-mail: richard.evans@csiro.au or helmut.thissen@csiro.au

Received 19 June 2015; revised 13 August 2015; accepted 16 September 2015 
a

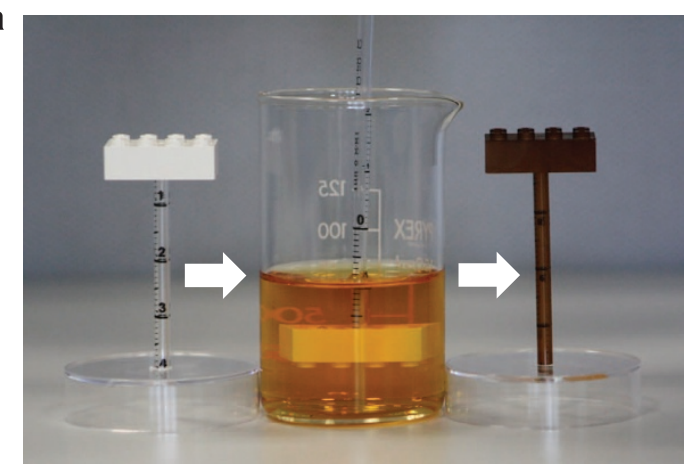

b

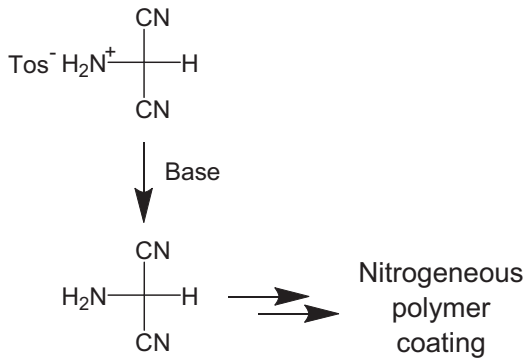

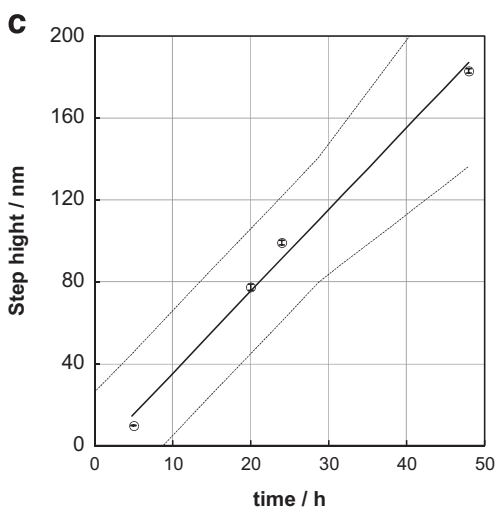

e

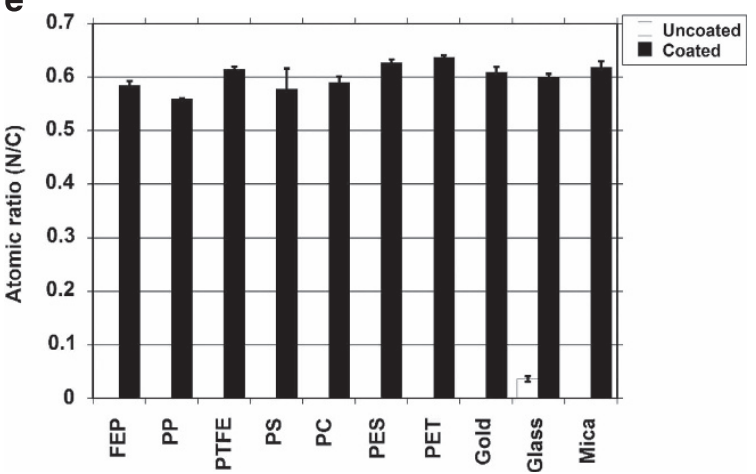

d

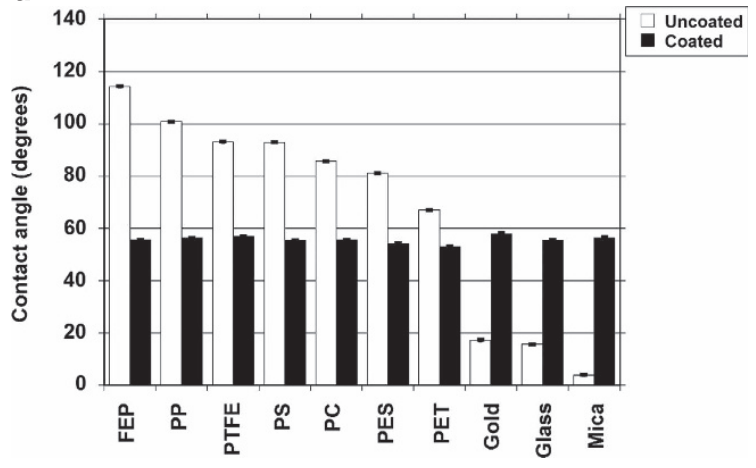

f

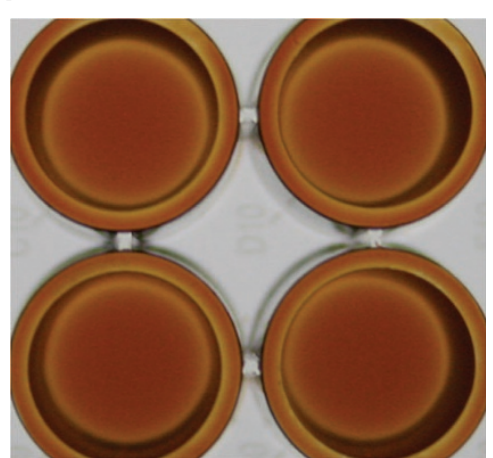

Figure 1 Polymers inspired by prebiotic chemistry and surface characterization. (a) Aminomalononitrile (AMN)-based coatings can be formed in a simple onestep procedure. (b) These coatings are formed by neutralization of the AMN toluene sulfonic acid salt in aqueous solutions. (c) Thickness of AMN-based coatings as a function of deposition time determined by atomic force microscopy (AFM) section analysis on mica substrates ( $n=9$, error bars represent s.d.; lines are drawn to guide the eye). (d, e) These coatings can be deposited on a broad range of materials, reflected for example by the average water contact angles and the average N/C elemental ratios obtained from XPS spectra before and after coating for $24 \mathrm{~h}$. (f) AMN coatings display a distinctive brown color (example deposited for $24 \mathrm{~h}$ in individual wells of a 96 -well plate). N/C, nitrogen-to-carbon ratio; XPS, X-ray photoelectron spectroscopy.

biomedical applications such as cell culture tools, providing high cell attachment and surface coatings on implantable medical devices providing enhanced tissue integration.

\section{MATERIALS AND METHODS}

\section{General method for AMN-coating deposition}

The coating process has been optimized over the course of this project, which was based on our discovery that thickness and morphology are highly dependent on the following factors: (1) concentration of AMN, (2) buffer concentration and (3) temperature. According to our X-ray photoelectron spectroscopy (XPS) analyses, however, the chemistry of the coating and therefore all subsequent chemical manipulations do not depend on these factors. Please see the Supplementary Information for a detailed description of the protocol. As a general method, AMN p-toluenesulfonate (AMN) is dissolved in a phosphate-buffered solution (either PBS or pure phosphate), and the $\mathrm{pH}$ is adjusted to 8.5 to initialize the polymerization. Samples are incubated in this solution facing downwards to prevent deposition of precipitate that forms over the course of the treatment. After up to $48 \mathrm{~h}$, the samples were rinsed five times with MilliQ water (Millipore, Billerich MA, USA) and then incubated overnight in MilliQ water before being air dried in a laminar flow cabinet. The presence of the adherent coating was indicated by a light brown color change.

\section{Labeling and co-deposition with TFEA and TFAEH}

Labeling and co-deposition using 2,2,2-trifluoroethylamine (TFEA) and trifluoroacetaldehyde ethyl hemiacetal (TFAEH) were carried out using untreated 
Costar flat-bottom 96-well polystyrene plates (Corning, Corning, NY, USA) as a substrate. Labeling experiments were carried out after deposition of an AMN coating using $100 \mathrm{~mm}$ phosphate buffer at $\mathrm{pH} 8.5$ (see Supplementary Information). For the labeling experiments, a $1 \mathrm{~m}$ stock solution of the respective label at $\mathrm{pH} 8.5$ was prepared using $100 \mathrm{~mm}$ phosphate buffer. A 10 -fold serial dilution (6 steps) was then prepared to provide 7 concentrations ranging from $1 \mu \mathrm{M}$ to $1 \mathrm{M}$. Using these solutions and pure buffer as a control, eight wells per concentration were incubated for $24 \mathrm{~h}$. The wells were then washed three times with MilliQ water by pipetting and soaked in water for an additional $24 \mathrm{~h}$ before drying. For co-deposition experiments, a $2 \mathrm{~m}$ solution of the TFEA or TFAEH, respectively, was prepared and diluted to give seven concentrations between $2 \mu \mathrm{M}$ and $2 \mathrm{M}$ with a pH of 8.5. Simultaneously, a solution of AMN was prepared as described above and mixed at a ratio of 1:1 with the different label concentrations. These solutions were then used to coat eight wells per concentration by applying the coating and washing process as described above

\section{Surface modification with 4-bromobenzyl bromide (BBB)}

AMN coatings were deposited onto glass cover slip samples. Subsequently, the samples were incubated for $16 \mathrm{~h}$ in a solution of $100 \mathrm{mg}$ of BBB and $200 \mu \mathrm{l}$ of $0.1 \mathrm{M}$ triethylamine in $5 \mathrm{ml}$ of acetonitrile at $37^{\circ} \mathrm{C}$, followed by extensive repeated washing in acetonitrile and subsequently MilliQ water and drying in a laminar flow cabinet. Control samples were prepared under identical conditions using $100 \mathrm{mg}$ of 1,4-dibromobenzene instead of BBB.

\section{Metallization of AMN coatings}

Incorporation of silver was achieved by incubating the coated surfaces in the dark with aqueous solutions of silver nitrate (Chem-Supply Pty, Gillman, SA, Australia), which were prepared from a fresh $100 \mathrm{~mm}$ stock by 10 -fold serial dilution to give a range of concentrations between 1 and $100 \mathrm{~mm}$. A surface incubated with pure water served as a control. After $24 \mathrm{~h}$, the solutions were removed, and each well was washed three times with water using a pipette. The wells were then completely filled with water and left to soak for another $24 \mathrm{~h}$. After drying in a laminar flow chamber, samples for XPS were taken and then the plates were double sealed in plastic bags and $\gamma$-sterilized $(25 \mathrm{kGy}$, Steritech Pty, Dandenong VIC, Australia). To evaluate the relationship between silver content and thickness, coatings of different thicknesses including an uncoated control surface were incubated with $100 \mathrm{~mm}$ silver nitrate solutions following the same protocol.

\section{Deposition of coatings using $\mathrm{KCN}$}

Equimolar amounts of potassium cyanide $(65.1 \mathrm{mg}, \mathrm{KCN})$ (caution) and ammonium chloride $(53.5 \mathrm{mg}$ ) were dissolved in $2 \mathrm{ml}$ of MilliQ water. Glass samples were incubated in this solution at room temperature for 7 days. Subsequently, samples were washed five times with MilliQ water and then incubated overnight in MilliQ water before being air dried in a laminar flow cabinet. The presence of the coating was indicated by a light brown color change.

\section{X-ray photoelectron spectroscopy}

XPS analysis was performed using an AXIS HSi and AXIS Ultra DLD spectrometer (Kratos Analytical, Manchester, UK) with a monochromated Al $\mathrm{K} \alpha$ source at a power of $144 \mathrm{~W}(12 \mathrm{kV} \times 12 \mathrm{~mA})$ and $112 \mathrm{~W}(8 \mathrm{kV} \times 14 \mathrm{~mA})$, respectively, a hemispherical analyzer operating in the fixed analyzer transmission mode and the standard aperture (analysis area: $0.3 \times 0.7 \mathrm{~mm}$ ). Survey spectra were acquired at a pass energy of $160 \mathrm{eV}$. High-resolution spectra were recorded from individual peaks at $40 \mathrm{eV}$ pass energy.

\section{Atomic force microscopy experiments}

A Bruker FastScan atomic force microscope employing an Icon scanner head with NanoScope 9.0 software was used to measure surface topography in tapping mode with ultrasharp silicon tips (TAP150A, Bruker Corporation, Billerica, MA, USA). The tips used in this study have a typical force constant of $5 \mathrm{~N} \mathrm{~m}^{-1}$ and a resonant frequency of $150 \mathrm{kHz}$. Scans were performed at $0.8 \mathrm{~Hz}$ and 512 data points per scan line. All images were processed using NanoScope Analysis 1.5 software (Bruker Corporation)

\section{SEM analysis}

For the SEM analysis, samples were mounted on an aluminum stub with double-sided conductive carbon tape. These samples were then iridium coated using a Polaron SC5750 sputter coater (Quorum Technologies, Laughton, UK). The thickness of the iridium coating was $\sim 10 \mathrm{~nm}$. Once coated, the samples were placed into a Philips XL30 Field Emission SEM (Philips, Amsterdam, Netherlands) for imaging. An accelerating voltage of $2 \mathrm{kV}$ was used to obtain images. The image analysis software used was AnalySIS, developed by Olympus Soft-Imaging Solutions (Tokyo, Japan).

\section{Contact angle measurements}

Static contact angles were measured using an automated contact angle goniometer (KSV Instruments, Helsinki, Finland). A droplet with a volume of $\sim 5 \mu \mathrm{l}$ of ultrapure MilliQ water was used for the experiments. Average contact angles were calculated from 10 measurements, with each measurement recording the contact angles on both sides of the droplet.

\section{Cell culture experiments}

L929 mouse fibroblasts (cell line ATCC-CCL-1, Rockville, MD, USA, recently authenticated and tested for mycoplasma contamination by PCR assay at the Victorian Infectious Diseases Reference Laboratory, VIDREL) were used to investigate the cellular response to AMN-coated surfaces in comparison to tissue culture polystyrene and ultra-low attachment (ULA) control surfaces. Cells were cultured in minimum essential medium containing $10 \%$ fetal bovine serum and $1 \%$ nonessential amino acids (Invitrogen, Carlsbad, CA, USA). After cell seeding at a density of $2.5 \times 10^{4}$ cells per $\mathrm{cm}^{2}$, samples were incubated for $24 \mathrm{~h}$ at $37^{\circ} \mathrm{C}$ in humidified air containing $5 \% \mathrm{CO}_{2}$. Subsequently, substrates were washed once with minimum essential medium before a solution consisting of $100 \mu \mathrm{l}$ of phenazine methosulfate solution $\left(0.92 \mathrm{mg} \mathrm{ml}^{-1}\right.$ in DPBS), $2 \mathrm{ml}$ of 3-(4,5-dimethylthiazol-2-yl)-2,5-diphenyltetrazolium bromide (MTS) solution $\left(2 \mathrm{mg} \mathrm{ml}^{-1}\right.$ in DPBS) and $10 \mathrm{ml}$ of minimum essential medium was added. The substrates were incubated for $3 \mathrm{~h}$ at $37^{\circ} \mathrm{C}$ in humidified air containing 5\% $\mathrm{CO}_{2}$ before a colorimetric readout was performed at 490 and $655 \mathrm{~nm}$.

\section{Spatial control over cell attachment}

Samples with a size of $\sim 4 \mathrm{~cm}^{2}$ were cut out of six-well ULA plates and served as a substrate for this experiment. AMN was dissolved in PBS with a $\mathrm{pH}$ of 7.4. To induce polymerization, the $\mathrm{pH}$ was then adjusted to 8.5 using $0.1 \mathrm{M}$ sodium hydroxide, resulting in a final AMN concentration of $2 \%(\mathrm{w} / \mathrm{v})$. After $30 \mathrm{~min}$ of incubation, $100 \mu$ l of this solution was transferred to individual wells of a 96-well plate, which was used as a source plate in a microarray printer (NanoPrint LM60 microarrayer, ArrayIt, Sunnyvale, CA, USA). After contact printing of the solution onto the ULA-coated samples in an array pattern using pins with a diameter of $375 \mu \mathrm{m}$, samples were air dried in a laminar flow cabinet for $48 \mathrm{~h}$. Subsequently, samples were washed three times with MilliQ water before again being air dried in a laminar flow cabinet. Prior to cell seeding, samples were subjected to $\gamma$-sterilization at a dose of $15 \mathrm{kGy}$. L929 mouse fibroblasts were cultured on these substrates in minimum essential medium containing $10 \%$ fetal bovine serum and $1 \%$ nonessential amino acids. After cell seeding at a density of $2.5 \times 10^{4}$ cells per $\mathrm{cm}^{2}$, samples were incubated for $16 \mathrm{~h}$ at $37^{\circ} \mathrm{C}$ in humidified air containing $5 \% \mathrm{CO}_{2}$. After washing samples with medium, a Molecular Probes (Eugene, OR, USA) live/dead assay was performed, which resulted in live, attached cells being stained green and dead, and attached cells being stained red. Samples were then imaged using a Nikon TE 2000 Fluorescent Microscope (Tokyo, Japan).

\section{Evaluation of cytotoxicity}

The cytotoxicity of AMN coatings was evaluated using AMN coatings deposited on 24-well plates (TCPS Nunclon Delta Surface, Nunc, Roskilde, Denmark) according to the ISO, the International Organization for Standardization, Geneva, Switzerland. ISO 10993-12:2012 (Biological evaluation of medical 
devices-Part 12: Sample preparation and reference materials) and ISO 10993 5:2009 (Biological evaluation of medical devices-Part 5: Tests for in vitro cytotoxicity).

\section{Biofilm cultivation and quantitative determination}

Two biofilm-forming reference bacterial strains-Staphylococcus epidermidis RP62a (ATCC 35984) and Pseudomonas aeruginosa (ATCC 27853)—were used in this study. Bacterial stocks (stored at $-80^{\circ} \mathrm{C}$ in nutrient broth with $15 \%$ glycerol) were streaked onto nutrient agar (Oxoid, Basingstoke, UK) plates for use as the working stock. Bacterial biofilm culture was set up using a modification of a previously established method. ${ }^{31}$ Briefly, an overnight bacterial culture grown in nutrient broth was diluted 1:100 into biofilmspecific growth media, including tryptic soya broth for S. epidermidis (Oxoid) and Luria-Bertani broth for $P$. aeruginosa. One hundred microliters of the diluted bacterial suspensions was pipetted into a well in 96-well flat-bottom polystyrene microplates that had received different coating treatments and were incubated for $24 \mathrm{~h}$ at $37^{\circ} \mathrm{C}$ with gentle agitation (75 r.p.m.). After overnight incubation, the cell suspensions were aspirated and the wells were rinsed twice with $110 \mu \mathrm{l}$ of PBS per well to remove non-adherent cells. To quantify biofilms formed on surfaces of different treatments, the plate containing biofilms was heat-fixed in a $60^{\circ} \mathrm{C}$ oven for $1 \mathrm{~h}$ and then stained with $110 \mu \mathrm{l}$ of $1 \%(\mathrm{~W} / \mathrm{V})$ crystal violet for $10 \mathrm{~min}$. The crystal violet solution in the wells was then discarded, and the plates were washed four times to remove excess stains by submerging them in tubs of clean water. The plates were gently tapped on paper towels to remove excess water in wells. Two hundred microliters of $95 \%$ ethanol and 5\% acetic acid were added into each well and incubated at room temperature for $15 \mathrm{~min}$. One hundred microliters of solution from each well was transferred to a new plate. The amount of biofilm formed in each well was determined by reading its optical density with a Tecan Infinite M200 Plate Reader (Tecan, Maennedorf, Switzerland) at $600 \mathrm{~nm}$.
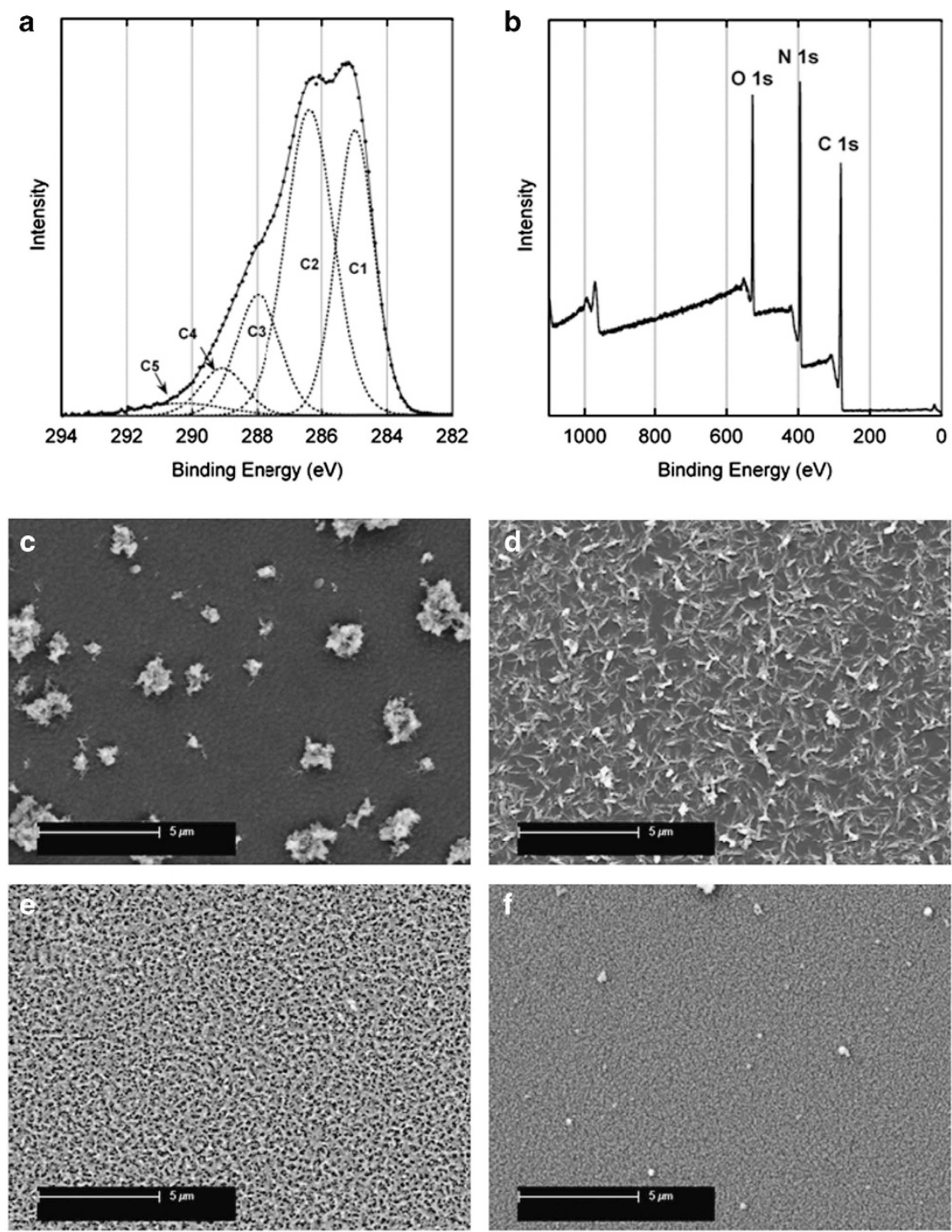

Figure 2 Surface characterization. (a) The different structural elements (annotated as components $\mathrm{C} 1-\mathrm{C} 5$, with $\mathrm{C} 1$ : $\mathrm{C}-\mathrm{H}, \mathrm{C}-\mathrm{C} ; \mathrm{C} 2$ : $\mathrm{C}-\mathrm{N}, \mathrm{C}=\mathrm{N}, \mathrm{C} \equiv \mathrm{N}, \mathrm{C}-\mathrm{O}$; $\mathrm{C} 3:(\mathrm{N}-) \mathrm{C}=\mathrm{O}, \mathrm{N}-\mathrm{C}-\mathrm{O} ; \mathrm{C} 4: \mathrm{O}-\mathrm{C}=\mathrm{O}$ and $\mathrm{C} 5$ : shake-up associated with unsaturated and/or aromatic hydrocarbon) that are present in $\mathrm{AMN}-\mathrm{based}$ polymer coatings are reflected in the C 1s high resolution XPS spectrum obtained on a polystyrene (PS) substrate. (b) The XPS survey spectrum obtained on the same sample highlights the characteristic high nitrogen content of AMN coatings. (c, d) SEM images obtained on mica and polystyrene surfaces coated over $24 \mathrm{~h}$ using a $100 \mathrm{~mm}$ phosphate buffer suggest a substrate-dependent coating morphology. (d-f) SEM images obtained on polystyrene surfaces coated over $24 \mathrm{~h}$ using $100 \mathrm{~mm}$ phosphate buffer, $10 \mathrm{~mm}$ phosphate buffer and no buffer, respectively, suggesting a phosphate concentration-dependent coating morphology. AMN, aminomalononitrile; XPS, X-ray photoelectron spectroscopy. 


\section{RESULTS AND DISCUSSION}

We examined the suitability of the AMN polymerization process for the formation of coatings on a range of substrate materials. Typical conditions that yield films suitable for many applications in materials science, including biomedical applications, are a buffered solution containing $2 \%(\mathrm{w} / \mathrm{v})$ of $\mathrm{AMN}$, adjusted to a $\mathrm{pH}$ of 8.5 . This results in the formation of an adherent brown AMN coating on materials incubated in that solution for up to $48 \mathrm{~h}$ under ambient atmospheric conditions at room temperature. The coatings are sufficiently robust to withstand the subsequent manipulations described herein. Atomic force microscopy section analysis demonstrated that the coating thickness can be controlled via deposition time and that a coating thickness of up to $\sim 200 \mathrm{~nm}$ can be achieved within $48 \mathrm{~h}$ of incubation (Figure 1c, Supplementary Figures S2 and S3).

Importantly, the AMN coating can be deposited on a broad range of substrates, including polymers, metals and other inorganic materials and that the physicochemical properties are independent of the underlying surface chemistry. The water contact angles as well as atomic nitrogen-to-carbon ratio (N/C) ratios determined by XPS after a deposition time of $24 \mathrm{~h}$ are practically identical across all substrates tested here (Figures 1d and e,Supplementary Tables S1 and S2).

Because AMN polymer also precipitates from solution during coating formation, samples used in this study were placed face down during the polymerization process to minimize settling of precipitate on the substrate surface. This coating strategy is suitable for more complex substrate geometries, including porous materials, microfluidic devices and multi-well plates (Figure 1f), which can be readily coated for subsequent use, for example, in cell culture applications.

The precise structure of the polymers obtained from HCN and its oligomers has remained ambiguous despite many years of meticulous research, reflecting their complex nature (Supplementary Figure S1). It is beyond the scope of this report to characterize the coatings further. However, XPS C 1s high resolution spectra of deposited coatings and the components that can be fitted for these spectra (Figure 2a) are consistent with the structural elements that have been proposed for $\mathrm{HCN}$-derived polymers. ${ }^{32-34}$ The XPS survey spectrum (Figure $2 \mathrm{~b}$ ) also reflects the unusually high $\mathrm{N} / \mathrm{C}(\mathrm{N} / \mathrm{C}=0.61 \pm 0.02)$, which is characteristic for these coatings. In comparison, common nitrogenous polymers used in biomedical applications such as polyethyleneimine, poly(L-lysine) and polyallylamine have significantly lower N/C ratios of $0.5,0.33$ and 0.33 , respectively.

The morphology of the polymer coatings appears to be influenced by the substrate material. SEM images obtained after deposition of the coating on mica (Figure 2c) and polystyrene (Figure 2d) show embedded particles within an otherwise homogenous coating. The morphology observed on polystyrene (Figure 2d) in particular may be of interest in applications requiring a high surface area. SEM images of coatings deposited on polystyrene using $100 \mathrm{~mm}$ phosphate buffer (Figure 2d), $10 \mathrm{~mm}$ phosphate buffer (Figure 2e) and water adjusted to $\mathrm{pH} 8.5$ with $\mathrm{NaOH}$ (Figure 2f) indicate that the morphology of polymer coatings is also influenced by the buffer concentration used. All SEM images, however, suggest the formation of pinhole-free coatings. This observation is consistent with our XPS and atomic force microscopy data (Supplementary Tables S2, Supplementary Figure S2).

We also explored the rich chemistry known to be present in AMN polymers for potential further functionalization by either copolymerization during coating deposition or alternatively reaction with the coating subsequent to deposition. The coating formation is a selfpolymerization reaction potentially involving active methylenes and primary amines attacking reactive nitrile groups to form an amidine. Other primary amines, as well as reactive groups such as aldehydes, were therefore expected to be either incorporated into the polymer coating when present in the aqueous polymerization solution as a comonomer or incorporated into the polymer coating when reacted after coating deposition. For example, the reaction with primary amines was demonstrated by the incorporation of 2,2,2-TFEA either during
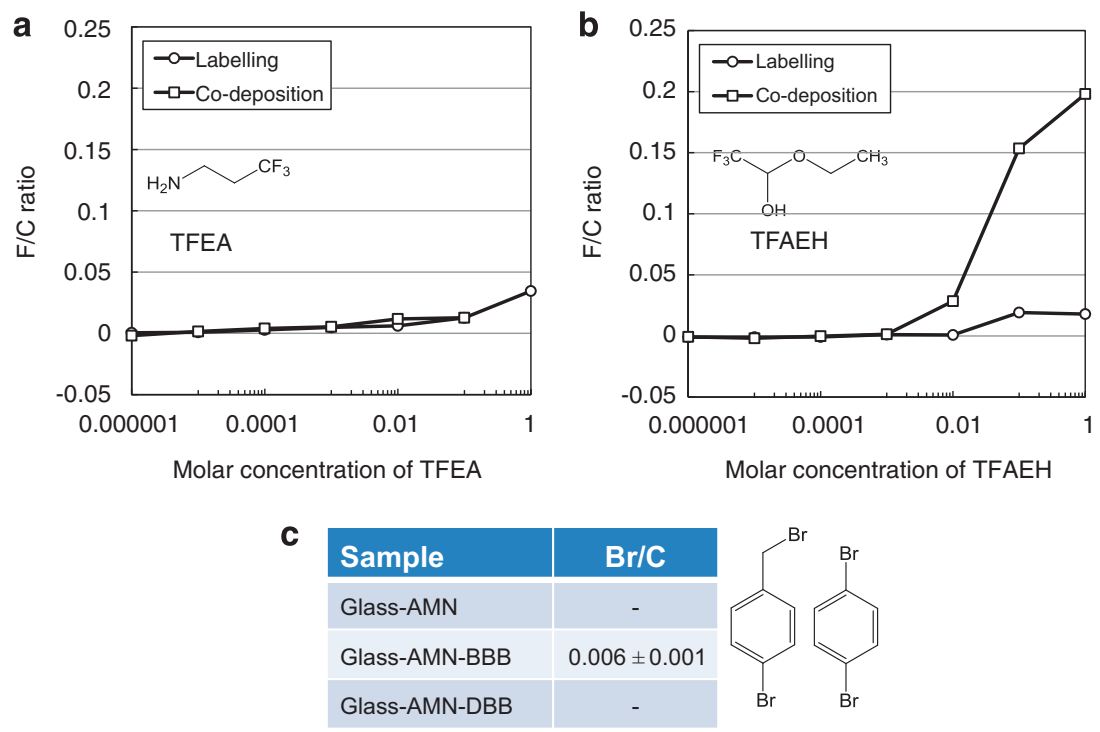

Figure 3 Co-deposition and labeling. (a) The incorporation of molecules carrying amine functional groups into AMN coatings during deposition and postdeposition labeling was demonstrated using the XPS label TFEA. (b) The incorporation of aldehydes, exemplified by the XPS label TFAEH, was demonstrated both during coating formation and post-deposition labeling, with greater incorporation of the aldehyde achieved during co-deposition ( $n=3$, error bars are the same size as markers). (c) The ability of AMN coatings to undergo subsequent reactions with an electrophile was exemplified using the XPS label BBB and the non-reactive control compound 1,4-dibromobenzene (DBB). AMN, aminomalononitrile; TFAEH, trifluoroacetaldehyde ethyl hemiacetal; TFEA, 2,2,2trifluoroethylamine; XPS, X-ray photoelectron spectroscopy. 
polymerization or in a post-deposition reaction illustrated by the detection of fluorine in the resulting coating using XPS (Figure 3a, Supplementary Figure S4, Supplementary Table S3). Because AMN possesses two nitrile groups for every amine, there is formally an excess of nitriles available for this reaction (Supplementary Figures S5 and S6). To explore the nucleophilic character of the coating, labeling with and co-deposition of TFAEH was carried out. Again, the incorporation of fluorine in the resulting coating was demonstrated using XPS analysis (Figure 3b, Supplementary Figure S7, Supplementary Table S4). Incorporation of aldehydes or their precursors may be rationalized by either imine formation with amines or active methylene groups reacting with aldehydes under basic conditions (Supplementary Figure S8). These results also suggest that the incorporation of aldehydes is more efficient during co-deposition as indicated by the higher atomic percentage of fluorine detected. The presence of amines within the AMN polymer also suggests reactivity towards electrophiles, as shown by the reaction with $\mathrm{BBB}$ and subsequent XPS analysis. A control experiment using an equivalent yet unreactive compound, $p$-dibromobenzene, indicates that the detection of bromine was not due to adsorption (Figure 3c).
The highly nitrogenous character of the AMN coatings was also expected to provide a suitable substrate for the complexation of metal ions. Because silver-containing coatings are of particular interest in antimicrobial surface applications, ${ }^{35}$ we intended to prove this concept by incubating $\mathrm{AMN}$ in aqueous silver nitrate solutions in the dark with concentrations spanning several orders of magnitude from $10^{-9}$ to $0.1 \mathrm{M}$. The metalized films contained both reduced and ionic silver as demonstrated by XPS (Figure 4a, Supplementary Table S5). The Ag MVV Auger region of the XPS spectra (Figure 4b) reveals that some of the silver in the coating is present in its reduced form on coatings that have been treated with higher silver nitrate concentrations $(0.01$ and $0.1 \mathrm{M})$. This emergence of metallic silver is not easily explained in the absence of complete structural knowledge of the coating. Unlike polydopamine-based coatings that provide a reductive chemistry, ${ }^{18}$ we have found no direct indication that our coating is involved in redox processes that may cause metallic silver to form on the surface. However, disproportionation reactions of silver, such as $2 \mathrm{Ag}^{+}+\mathrm{L} \rightarrow$ $\mathrm{AgL}^{2+}+\mathrm{Ag}(0)$, in the presence of certain ligands (L) have been reported. ${ }^{36}$ Our coating provides several electron donors such as amines and nitriles for metal coordination and may explain our
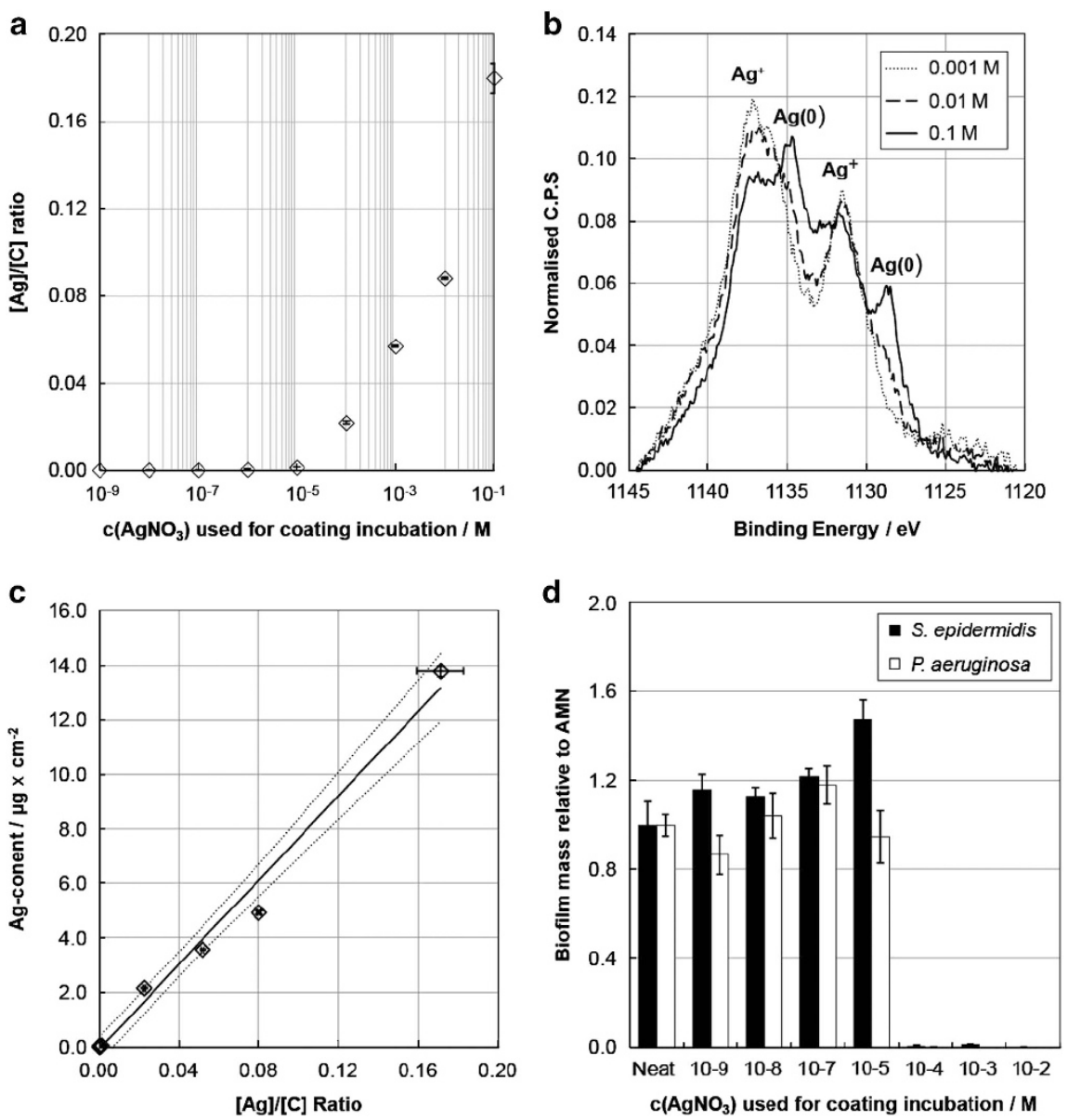

Figure 4 Surface analysis, quantification and biological evaluation of silver-treated AMN coatings. (a) Atomic Ag/C ratios derived from quantification of XPS survey spectra. (b) Auger spectra of three different surfaces with increasing silver surface density showing the increasing presence of metallic silver as the surface density increases. (c) Correlation of atomic Ag/C ratio determined by XPS with the absolute amount, if silver incorporated, determined by ICP-OES. (d) $24 \mathrm{~h}$ biofilm assay-based semi-quantitative crystal violet staining. The two representative bacterial strains, $S$. epidermidis (Gram-positive) and $P$. aeruginosa (Gram negative), show a concentration-dependent decrease in biofilm formation with biofilm mass for both strains sharply dropping of above concentrations of $10^{-5} \mathrm{M} \mathrm{AgNO}_{3}$ used for incubating the AMN coatings. AMN, aminomalononitrile; ICP-OES, inductively coupled plasma optical emission spectroscopy; XPS, X-ray photoelectron spectroscopy. 
observations. In addition to the relative quantification of silver by XPS, we determined the absolute amount of silver incorporated into the coating. To this end, nitric acid was used to dissolve the coating and all silver. The resulting solutions were analyzed by inductively coupled plasma optical emission spectroscopy (ICP-OES). The ICP-OES data, which represent the silver content of the entire coating, exhibit a good linear correlation (Figure 4c) with the XPS-based data, which under the experimental conditions used here represent the first 5 to $10 \mathrm{~nm}$ of the coating. The absolute amount of silver also shows a significant linear correlation with coating thickness (see Supplementary Information,Supplementary Figure S9). These results imply that silver nitrate completely penetrates the AMN coating and that silver is uniformly distributed. Both quantification methods detect silver on those surfaces treated with silver nitrate concentrations $\geqslant 10^{-5} \mathrm{M}$. Below this threshold, practically no silver is detected on the surface. It should be noted that the quantification of silver on all surfaces was carried out after a $24 \mathrm{~h}$ washing step, so these data do not represent the silver adsorption isotherms for this coating. However, the fact that after $24 \mathrm{~h}$ considerable amounts of silver are detected indicates that silver does bind to the polymer and that it is released slowly over a time period extending beyond $24 \mathrm{~h}$.

The silver-treated surfaces were also evaluated for their ability to prevent biofilm growth of two well-known biofilm-forming bacterial strains: S. epidermidis RP62A (a representative Gram-positive bacterium) and $P$. aeruginosa (a representative Gram-negative bacterium). ${ }^{37,38}$ To this end, a semi-quantitative standard biofilm assay based on crystal violet staining was performed $24 \mathrm{~h}$ after initial contact of the bacteria with the surfaces. As judged by optical density, no planktonic bacterial growth was observed above biofilm negative surfaces, suggesting release of the antimicrobial species. The results show a concentration-dependent anti-biofilm activity, with biofilm growth sharply dropping off on surfaces that had been treated with $\geqslant 10^{-4} \mathrm{M}$ silver nitrate (Figure $4 \mathrm{~d}$ ).

There are a number of routes into $\mathrm{HCN}$-derived polymers (Figure 5). The use of $\mathrm{AMN}$ as a polymer precursor is particularly convenient, especially for lab-scale preparations. However, a buffered solution of equimolar amounts of KCN (caution) and ammonium chloride also provided a light brown polymer coating analogous to that obtained from AMN (N/C $=0.65 \pm 0.1$, Supplementary Table S6). This route may have benefits in regard to a potential industrial scaleup of the coating technology.

Because prebiotic chemistry and polymers are considered to be important for the production of the molecules and macromolecules required for life to have arisen, it is surprising that prebiotic or prebiotic-inspired polymers have not previously been examined for their compatibility with biology, with the exception of a single study investigating the bacterial metabolism of tholins. ${ }^{39}$ We used L929 mouse fibroblasts to survey the properties of the AMN polymer after coating polystyrene multi-well plates and showed that the AMN coating provides outstanding cell attachment. When commercially available Corning ULA 96-well plates were coated with the AMN coating for $24 \mathrm{~h}$, cell attachment increased from $3 \pm 2 \%$ (ULA surface) to $127 \pm 5 \%$ (ULA-AMN surface) normalized against conventional tissue culture polystyrene plates $(100 \pm 13 \%)$ (Figures $6 \mathrm{a}-\mathrm{d}$,Supplementary Table S7). (Note that the pale brown color of the AMN coating does not interfere with spectroscopy and imaging.) Because the control over biointerfacial interactions is the key to a broad range of new and improved biomedical devices, we also aimed to demonstrate the versatility of AMN coatings in this context. As an example, spatial control over the biological response was achieved by depositing $375 \mu \mathrm{m}$ diameter AMN

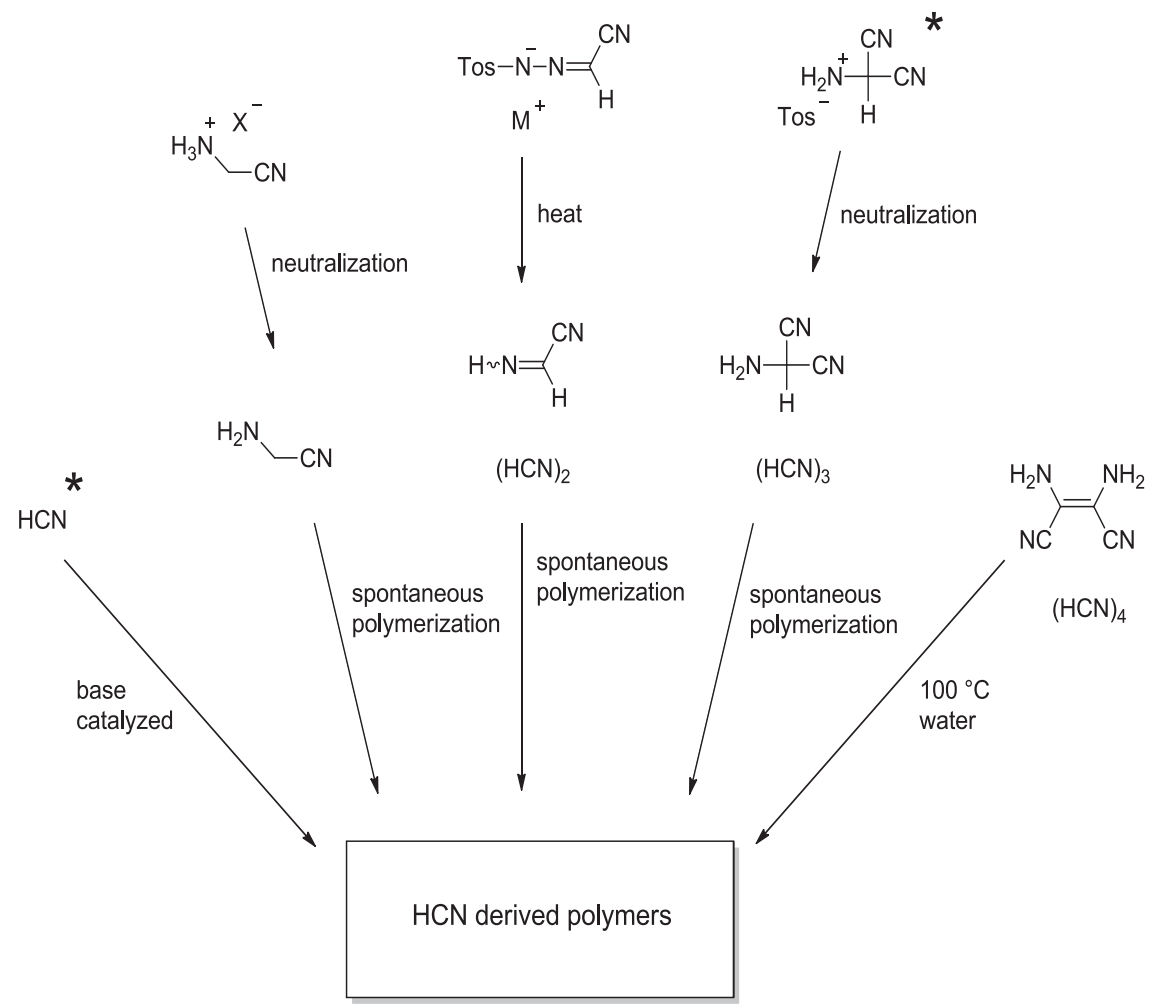

Figure 5 Routes for the formation of HCN-derived polymers. The reaction schematic illustrates the formation of HCN-derived polymers using different monomer units, including $\mathrm{HCN}$ itself, aminoacetonitrile $\mathrm{H}_{2} \mathrm{~N}-\mathrm{CH}_{2}-\mathrm{CN}$ (AAN), iminoacetonitrile (IAN), aminomalononitrile (AMN) and diaminomaleonitrile (DAMN). The routes explored in this study are highlighted $(*)$. HCN, hydrogen cyanide. 

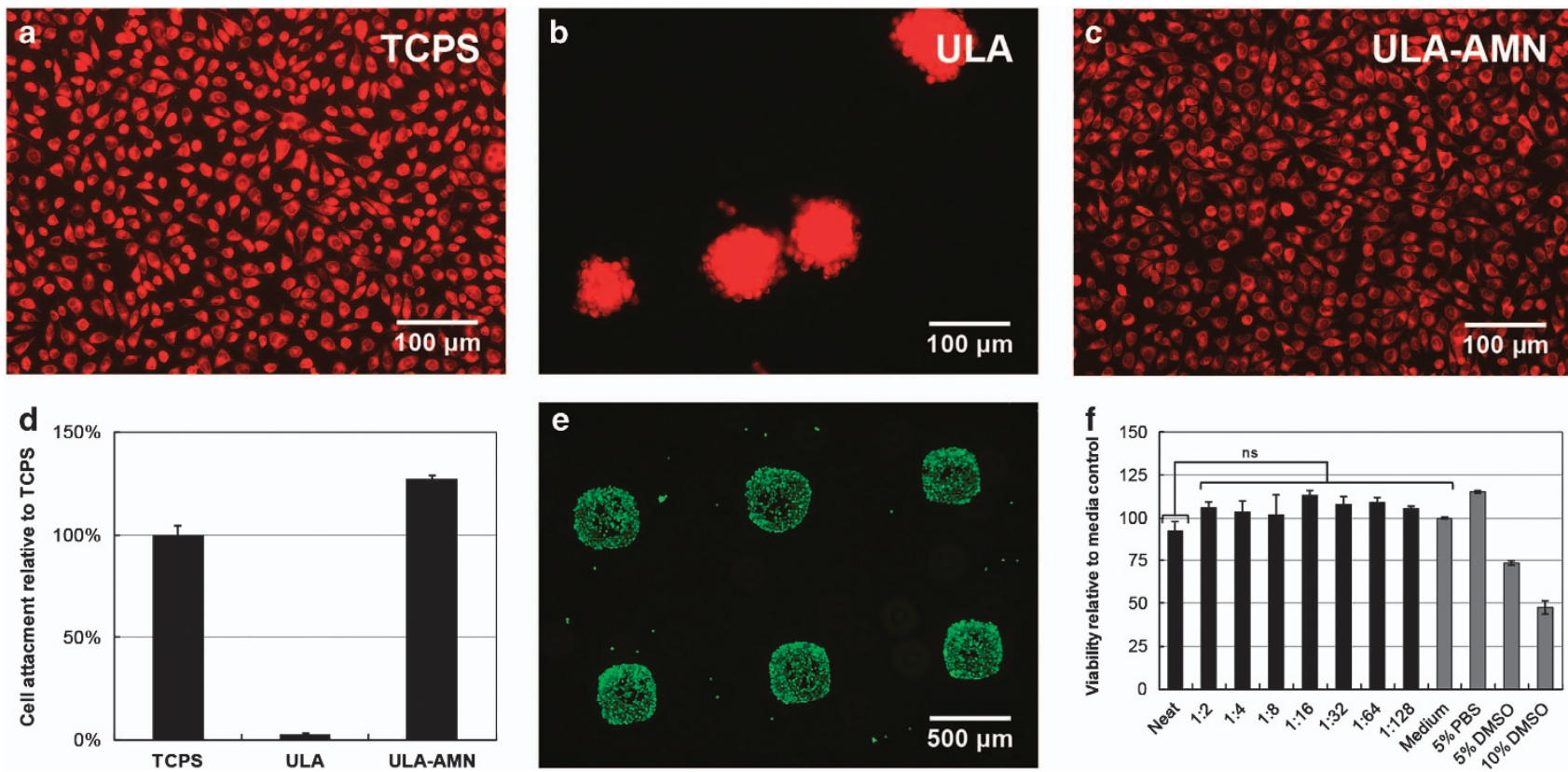

Figure 6 Biological response to AMN and silver-treated AMN coatings. (a-c) After $24 \mathrm{~h}$ culture, L929 mouse fibroblast cells show excellent attachment and spreading on tissue culture polystyrene (TCPS) control surfaces (a), while attachment is prevented on Corning Ultra-Low Attachment (ULA) control surfaces, leading to cell clumping (b). On AMN polymer coatings deposited onto ULA surfaces (ULA-AMN), cell attachment and spreading is equivalent to the TCPS surface, reversing the effect of the underlying ULA surface (c). (d) The quantification of cell attachment via an MTS assay supports these observations ( $n=8$, error bars represent s.e.m.). (e) Spatial control over L929 cell attachment was demonstrated on an ULA surface onto which an AMN solution was microarray contact printed. (f) Toxicity of a twofold serial dilution of AMN surface extracts and control standards against L929 mouse fibroblasts according to ISO 10993-12:2002(E) and ISO 10993-5:2009(E) ( $n=4$ for media, PBS and DMSO controls, $n=3$ for neat AMN and dilutions). No statistically significant difference was observed between the AMN extract, its dilutions and the media control (ANOVA single factor analysis, $P=0.29$ ). AMN, aminomalononitrile; ANOVA, analysis of variance; DMSO, dimethyl sulfoxide; NS, not significant; PBS, phosphate-buffered solution.

coatings onto ULA substrates using a micro-contact printer. The subsequent seeding of L929 mouse fibroblasts demonstrated that cell attachment was restricted to areas coated with the AMN polymer (Figure 6e). Importantly, no cytotoxicity was observed during extraction experiments using AMN-coated substrates carried out according to ISO norm protocols (Figure 6f). The lack of cytotoxicity and the celladhesive nature of AMN coatings underline their suitability in a broad range of biomedical applications. It would appear that prebiotic polymers may not only have provided the building blocks for the origin of life but also provided an excellent substrate for it.

There is enormous scope for re-examining 60 years of prebiotic chemical research from the perspective of its application to polymer, material and life sciences. The focus of prebiotic chemistry has been the understanding of the chemistry that eventually led to life. We have shown that coatings inspired by such chemistry may now be adapted and practically exploited in other disciplines. Furthermore, we have developed a new surface modification method that joins the very few generic aqueous coating methodologies available. The simplicity of this method, combined with the mild conditions required, point to a wide variety of potential applications.

\section{CONFLICT OF INTEREST}

The authors declare no conflict of interest.

\section{ACKNOWLEDGEMENTS}

We thank Thomas Gengenbach (CSIRO) for assistance with regard to the interpretation of XPS spectra, as well as Jacinta White and Veronica Glattauer (CSIRO) for assistance with regard to cell culture experiments. We also thank Yeşim Gözükara and Winston Liew (CSIRO) for assistance with the ICP-OES measurements. We acknowledge the support of a National Health \& Medical Research Council Program grant (606788). TL is an ARC Australian Laureate Fellow (FL130100038).

1 Lemmon, R. M. Chemical evolution. Chem. Rev. 70, 95-109 (1970)

2 Lambert, J.-F., Sodupe, M. \& Ugliengo, P. Prebiotic chemistry. Chem. Soc. Rev. 41 5373-5374 (2012).

3 Bada, J. L. New insights into prebiotic chemistry from Stanley Miller's spark discharge experiments. Chem. Soc. Rev. 42, 2186-2196 (2013).

4 The ascent of molecules. Nat. Chem. 5, 349-349 (2013).

5 Miller, S. L. A production of amino acids under possible primitive earth conditions. Science 117, 528-529 (1953)

6 Bada, J. L. \& Lazcano, A. Prebiotic soup-revisiting the Miller experiment. Science 300, 745-746 (2003).

7 Matthews, C. N. \& Moser, R. E. Peptide synthesis from hydrogen cyanide and water Nature 215, 1230-1234 (1967).

8 Matthews, C. N. \& Moser, R. E. Prebiological protein synthesis. Proc. Natl Acad. Sci. USA 56, 1087-1094 (1966).

9 Matthews, C. N. \& Minard, R. D. Hydrogen cyanide polymers connect cosmochemistry and biochemistry. Proc. Int. Astron. Union 4, 453-458 (2008).

10 Matthews, C. N. \& Minard, R. D. Hydrogen cyanide polymers, comets and the origin of life. Faraday Discuss. 133, 393-401 (2006).

11 Ferris, J. P. \& Hagan, W. J. Jr. HCN and chemical evolution: The possible role of cyano compounds in prebiotic synthesis. Tetrahedron 40, 1093-1120 (1984).

12 Sanchez, R. A., Ferris, J. P. \& Orgel, L. E. Studies in prebiotic synthesis: II. Synthesis of purine precursors and amino acids from aqueous hydrogen cyanide. J. Mol. Biol. 30 223-253 (1967).

13 Ferris, J. P. \& Joshi, P. C. Chemical evolution from hydrogen cyanide: photochemical decarboxylation of orotic acid and orotate derivatives. Science 201, 361-362 (1978).

14 Sagan, C. \& Khare, B. N. Tholins: organic chemistry of interstellar grains and gas. Nature 277, 102-107 (1979).

15 Khare, B. N., Sagan, C., Thompson, W. R., Arakawa, E. T., Meisse, C. \& Tuminello, P. S. Optical properties of poly-HCN and their astronomical applications. Can. J. Chem. 72 678-694 (1994). 
16 Raulin, F., Brasse, C., Poch, O. \& Coll, P. Prebiotic-like chemistry on Titan. Chem. Soc. Rev. 41, 5380-5393 (2012).

17 Castner, D. G. \& Ratner, B. D. Biomedical surface science: foundations to frontiers Surface Sci. 500, 28-60 (2002).

18 Lee, H., Dellatore, S. M., Miller, W. M. \& Messersmith, P. B. Mussel-inspired surface chemistry for multifunctional coatings. Science 318, 426-430 (2007).

19 Decher, G. Fuzzy nanoassemblies: toward layered polymeric multicomposites. Science 277, 1232-1237 (1997).

20 Thissen, H., Evans, R. A. \& Koegler, A. Hydrogen cyanide-based polymer surface coatings and hydrogels. WO 2013170308-A1 (2013).

21 Sanchez, R., Ferris, J. \& Orgel, L. E. Conditions for purine synthesis: did prebiotic synthesis occur at low temperatures? Science 153, 72-73 (1966).

22 Freeman, F. Reactions of malononitrile derivatives. Synthesis 1981, 925-954 (1981).

23 Evans, R. A., Lorencak, P., Ha, T. K. \& Wentrup, C. HCN dimers: iminoacetonitrile and N-cyanomethanimine. J. Am. Chem. Soc. 113, 7261-7276 (1991).

24 Moser, R. E., Claggett, A. R. \& Matthews, C. N. Peptide formation from aminomalononitrile (HCN trimer). Tetrahedron Lett. 9, 1605-1608 (1968).

25 Matthews, C. N. in Origins: Genesis, Evolution and Diversity of Life Vol. 6: (ed. Seckbach, J.) 121-135 (Kluver Academic Publishers, Dordrecht, Netherlands, 2004).

26 Mamajanov, I. \& Herzfeld, J. HCN polymers characterized by solid state NMR: Chains and sheets formed in the neat liquid. J. Chem. Phys. 130, 134503 (2009).

27 Umemoto, K., Takahasi, M. \& Yokota, K.Studies on the structure of HCN oligomers. Orig. Life 17, 283-293 (1987).

28 Oró, J. \& Kamat, S. S. Amino-acid synthesis from hydrogen cyanide under possible primitive earth conditions. Nature 190, 442-443 (1961)

29 Menor-Salván, C. \& Marín-Yaseli, M. R. Prebiotic chemistry in eutectic solutions at the water-ice matrix. Chem. Soc. Rev. 41, 5404-5415 (2012).

30 Ruiz-Bermejo, M., Zorzano, M.-P. \& Osuna-Esteban, S. Simple organics and biomonomers identified in HCN polymers: an overview. Life 3, 421-448 (2013).

31 Deighton, M. A., Capstick, J., Domalewski, E. \& van Nguyen, T. Methods for studying biofilms produced by Staphylococcus epidermidis. Methods Enzymol. 336, 177-195 (2001).
32 Ruiz-Bermejo, M., de la Fuente, J. L., Rogero, C., Menor-Salván, C., Osuna-Esteban, S. \& Martín-Gago, J. A. New insights into the characterization of 'insoluble black HCN polymers'. Chem. Biodivers. 9, 25-40 (2012).

33 de la Fuente, J. L., Ruiz-Bermejo, M., Menor-Salván, C. \& Osuna-Esteban, S. Thermal characterization of HCN polymers by TG-MS, TG, DTA and DSC methods. Polym. Degrad. Stab. 96, 943-948 (2011).

34 Andersen, J. L., Andersen, T., Flamm, C., Hancyz, M., Merkle, D., Stadler, P. F. Navigating the chemical space of HCN polymerization and hydrolysis: guiding graph Grammars by mass spectrometry data. Entropy 15, 4066-4083 (2013).

35 Maillard, J.-Y. \& Hartemann, P. Silver as an antimicrobial: facts and gaps in knowledge. Crit. Rev. Microbiol. 39, 373-383 (2013).

36 Kestner, M. O. \& Allred, A. L. Ligand-induced disproportionation of silver(I). J. Am. Chem. Soc. 94, 7189-7189 (1972).

37 Hogan, S., Stevens, N. T., Humphreys, H., O'Gara, J. P. \& O'Neill, E. Current and future approaches to the prevention and treatment of staphylococcal medical device-related infections. Curr. Pharm. Des. 21, 100-113 (2015).

38 Rasamiravaka, T., Labtani, Q., Duez, P. \& El Jaziri, M. The formation of biofilms by Pseudomonas aeruginosa: a review of the natural and synthetic compounds interfering with control mechanisms. Biomed Res. Int. 2015, 759348 (2015).

39 Stoker, C. R., Boston, P. J., Mancinelli, R. L., Segal, W., Khare, B. N. \& Sagan, C. Microbial metabolism of tholin. Icarus 85, 241-256 (1990).

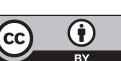

This work is licensed under a Creative Commons Attribution 4.0 International License. The images or other third party material in this article are included in the article's Creative Commons license, unless indicated otherwise in the credit line; if the material is not included under the Creative Commons license, users will need to obtain permission from the license holder to reproduce the material. To view a copy of this license, visit http:// creativecommons.org/licenses/by/4.0/

Supplementary Information accompanies the paper on the NPG Asia Materials website (http://www.nature.com/am) 